\title{
Use of Intravenous Immunoglobulin (Prevagen or Octagam) for the Treatment of COVID-19: Retrospective Case Series
}

\author{
Felix J.F. Herth ${ }^{\mathrm{a}}$ George Sakoulas $^{\mathrm{b}}$ Fadi Haddad $^{\mathrm{c}}$ \\ aDepartment of Pneumology and Critical Care Medicine, Thoraxklinik, and Translational Lung Research Center \\ Heidelberg, University of Heidelberg, Heidelberg, Germany; ${ }^{b}$ Sharp Memorial Hospital and the University of \\ California, San Diego, CA, USA; 'Sharp Grossmont Hospital, La Mesa, CA, USA
}

\section{Established Facts}

- Coronavirus disease 19 (COVID-19) cases continue to rise producing an unprecedented global health and economic crisis.

- The rapid progression of lung involvement in patients with severe COVID-19 makes it imperative to develop efficient strategies to potentially attenuate the need for mechanical ventilation.

\section{Novel Insights}

- Treatment with systemic immune modulators, such as intravenous immunoglobulin (IVIG), may attenuate aberrant immune responses, and the subsequent inflammatory responses, observed in the severe stages of acute respiratory distress.

- It may be useful to consider IVIG therapy to promote satisfactory recovery and reduce the burden of care for COVID-19 patients.

\section{Keywords}

Coronavirus disease $19 \cdot$ Acute respiratory distress syndrome $\cdot$ Intravenous immunoglobulin

\begin{abstract}
Treatment with immunomodulators, such as intravenous immunoglobulin (IVIG), may attenuate inflammatory responses observed in the severe stages of acute respiratory distress syndrome (ARDS) caused by coronavirus disease 19 (COVID-19). We retrospectively evaluated the clinical courses of 12 COVID-19 patients who received IVIG at various stag-
\end{abstract}

es of their illness, including within the first $72 \mathrm{~h}$ of clinical presentation, after initiation of mechanical ventilation, and after prolonged ventilation and ICU stay. The patients included 9 men and 3 women with a median age of 50 years (range 23-74), median Charlson Comorbidity Score of 2 (range 0-7), and median Acute Physiology and Chronic Health Evaluation Score of 13 (range 5-33) at the time of IVIG. The IVIG total dose ranged from 0.5 to $2.0 \mathrm{~g} / \mathrm{kg}$ (median $1.25 \mathrm{~g} / \mathrm{kg}$ ) distributed over 1-4 daily doses. The most common regimen received was $0.5 \mathrm{~g} / \mathrm{kg}$ daily for 3 days. The median time to IVIG administration was 9 days (range 0-48 days) after admission. The median time from first IVIG dose
(C) 2020 The Author(s)

Published by S. Karger AG, Basel

This is an Open Access article licensed under the Creative Commons Attribution-NonCommercial-4.0 International License (CC BY-NC) (http://www.karger.com/Services/OpenAccessLicense), applicable to the online version of the article only. Usage and distribution for commercial purposes requires written permission.
George Sakoulas

Biomedical Research Facility II, University of California at San Diego

Room 4114, 9500 Gilman Drive, Mail Code 0760

La Jolla, CA 92093-0760 (USA)

george.sakoulas@sharp.com 
administration to hospital discharge was 14 days (range 3-48). The 5 patients who received IVIG $\leq 4$ days of admission demonstrated a significantly shorter length of hospital stay after treatment (median 7 days, range 3-14 days) than the 7 patients who received it $>7$ days after admission (median 33 days, range 8-48 days, $p=0.03$, Mann-Whitney $U$ test). These cases demonstrate that IVIG may improve the clinical state of patients with moderate to severe COVID-19 infection. Despite very high illness severity scores, all patients survived hospital discharge. No thrombotic events occurred and IVIG was well tolerated, despite most cases demonstrating very elevated D-dimer suggestive of active intravascular fibrinolysis. We believe that IVIG warrants immediate clinical trial evaluation in COVID-19 to confirm its role as a mainstay treatment of moderate to severe COVID-19 infection as a means to reduce hospital stay and utilization of ICU resources, including mechanical ventilation, and potentially reduce mortality.

\footnotetext{
(C) 2020 The Author(s)

Published by S. Karger AG, Basel
}

\section{Introduction}

Cases of coronavirus disease 19 (COVID-19) continue to rise around the world, leading to unprecedented global health and economic crises [1,2]. Even regions that were highly successful in containing the spread of the virus are now seeing a resurgence of cases, indicating a tremendous medical need in vaccine prevention and more effective therapeutics. The ability to mitigate acute lung injury requiring supplemental oxygen and/or mechanical ventilation would be anticipated to not only improve patient outcomes but could potentially offload the tremendous burden on healthcare systems.

COVID-19 appears to represent yet another example of a classic biphasic infectious disease [3-6]. The first phase, driven by viral replication in the upper respiratory tract, is typically sub- or preclinical, marked by protean upper respiratory symptoms such as cough, pharyngitis, headache, fever, and myalgia. The unfortunate subset of patients who proceed to the second phase of illness driven by the adaptive immune response may experience severe morbidity including pneumonia, acute respiratory distress syndrome (ARDS), cardiomyopathy, endotheliitis, and multiple-organ failure [3-6]. Elevated serum concentrations of pro-inflammatory cytokines (tumor necrosis factor, interleukin-1 [IL-1], and IL-6) and chemokines (IL-8) point directly to a hyperinflammatory response, also known as a "cytokine storm," in the pathogenesis of the most clinically significant COVID-19 syndromes [7$11]$.

Intravenous immunoglobulin (IVIG) was first introduced in the USA for the treatment of primary immunodeficiencies in the 1980s but has increasingly been recognized for its anti-inflammatory and immunomodulating effects [12]. Approved IVIG preparations consist of highly purified immunoglobulins (Igs), mostly of the IgG class, obtained from thousands of screened donors [13]. The mechanism of action of IVIG is not completely understood, but it may modulate the immune response via multiple mechanisms including (i) inhibiting activation and functions of various monocytes, macrophages, dendritic cells, neutrophils, and natural killer cells in part by Fc-gamma receptor binding; (ii) neutralizing activated complement components and preventing membrane attack complex cellular killing; (iii) modulating B-cell functions and plasma cells; (iv) regulating T-cell balance between Treg cells and effector T cells (e.g., Th1, Th17); (v) downregulating the production of inflammatory cytokines $[8,12,14]$. Therefore, treatment with early systemic immune modulators, such as IVIG, may reduce aberrant immune responses, and the subsequent inflammatory responses, observed in the severe stages of ARDS [14].

Retrospective studies point to a potential benefit of IVIG treatment for COVID-19 ARDS in adults and the associated Kawasaki-like illness in children [15-22]. In anticipation of data on the use of IVIG in COVID-19 infection from a small pilot study and a larger, randomized trial currently underway, here, we present 12 patients (11 confirmed and 1 strongly suspected) with severe COVID-19 treated with IVIG in the USA and Germany.

\section{Study Methods}

A retrospective observational study was conducted using patient records from 2 hospitals in the USA (Sharp Grossmont Hospital in La Mesa, CA, USA; Sharp Memorial Hospital in San Diego, CA, USA) and 1 in Heidelberg, Germany (Thoraxklinik, Heidelberg University Hospital). The protocol was assessed by an Independent Review Board (IntegReview) in the USA and an Ethics Committee in Germany and was granted exempt status in both countries based on the nature of the retrospective evaluation. This study was conducted in compliance with all applicable regulatory guidelines. All study data were collected from preexisting patient records, which were de-identified.

Inclusion criteria included all hospitalized patients with confirmed/highly suspected COVID-19 and off-label treatment with IVIG that were not part of clinical trials of IVIG. As available, the following data parameters were evaluated: patient demographics, comorbidities and comorbidity index calculation (Charlson Co-
Herth/Sakoulas/Haddad 


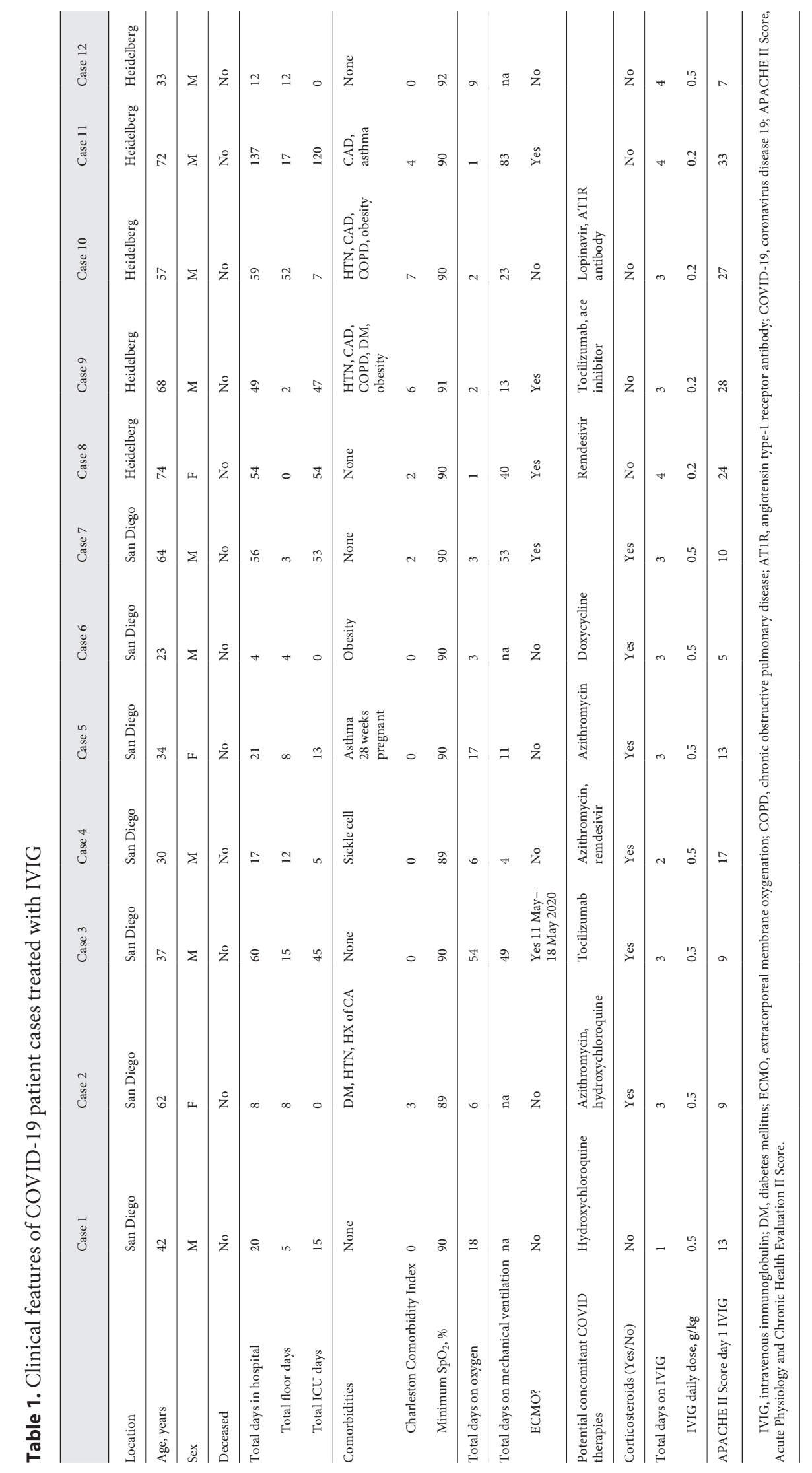



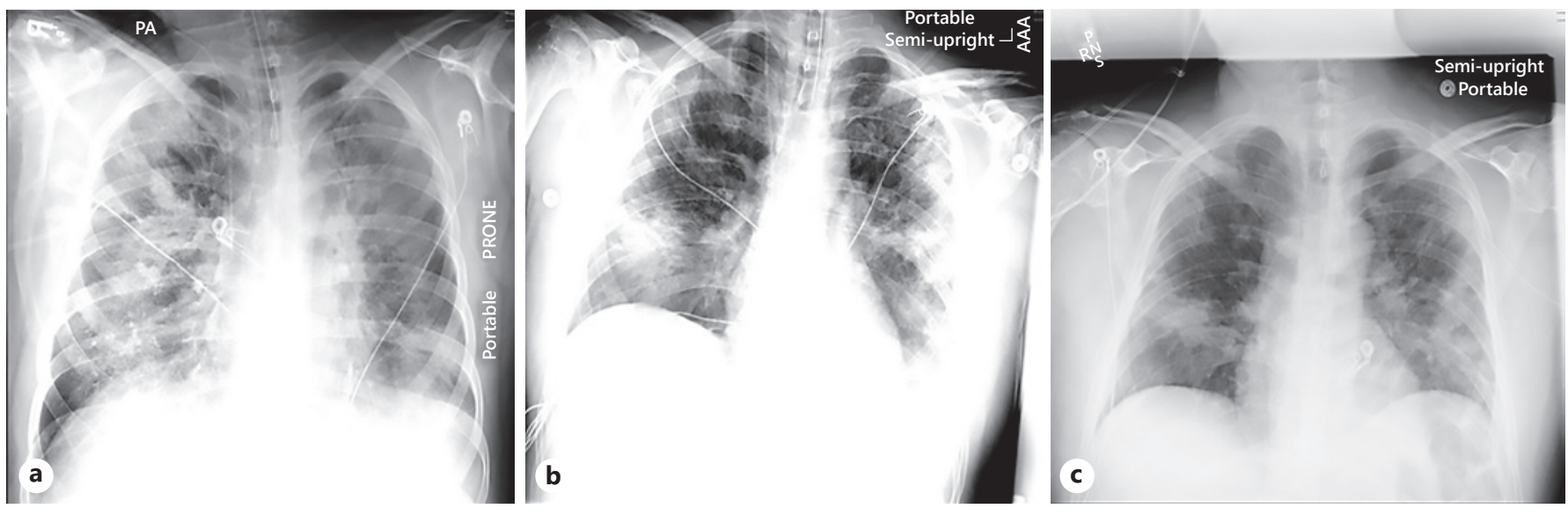

Fig. 1. Serial chest X-ray images from the COVID-19 patient described in case 1, demonstrating improvement on day 1 (a), day 5 (b), and day 8 (c) after administration of IVIG. COVID-19, coronavirus disease 19; IVIG, intravenous immunoglobulin.

morbidity Index), Acute Physiology and Chronic Health Evaluation Score (APACHE II Score), past medical history, vital signs, oxygen requirements, key laboratory/inflammatory biomarker evaluations, pertinent radiographs, days on mechanical ventilation, days in hospital (intensive care unit [ICU]/medical floor), IVIG daily dose and total dose, concomitant medications (including drugs [other than IVIG] for treatment of COVID-19), and adverse events.

\section{Case Summaries}

Between March and July 2020, 12 patients ( 9 men/3 women), with an average age of 50 years (range: $23-74$ years), met the criteria for inclusion in the case series. An overview of demographic information and case details are provided in Table 1.

\section{Case 1}

A 42-year-old male with no reported comorbidities presented the following onset of COVID-19 symptoms and a positive test. Following admission, he was initially given oxygen support. On hospital day 2, he was also given hydroxychloroquine for 7 days. Despite these interventions, the patient's condition deteriorated. He was moved to the ICU and intubated on day 5 . The patient continued to decline. On day 7, he was given IVIG for 1 day at 0.5 $\mathrm{g} / \mathrm{kg}$. Following IVIG administration, the patient's clinical course and chest X-ray improved (Fig. 1). He was extubated on day 18 and discharged from the hospital on day 20.

\section{Case 2}

A 62-year-old woman, with diabetes mellitus, hypertension, and history of breast cancer, presented following 5 days of coughing, fever, and an increase in dyspnea. She tested positive for COVID-19. She was admitted on room air but had rapidly worsening hypoxia over the next $72 \mathrm{~h}$ requiring up to $6 \mathrm{~L}$ of $\mathrm{O}_{2}$ via a nasal cannula, with development of infiltrates on chest X-ray (Fig. 2). Her fever increased to $39^{\circ} \mathrm{C} / 102.2^{\circ} \mathrm{F}$. On admission, she was given intravenous (IV) doxycycline and hydroxychloroquine. On hospital days $4-6$, she was given IVIG at $0.5 \mathrm{~g} / \mathrm{kg}$ per day premedicated with methylprednisolone $40 \mathrm{mg}$ IV. This was marked by a dramatic improvement in fever and oxygenation (Fig. 2). She was discharged on hospital day 8 on room air.

\section{Case 3}

A 37-year-old male patient, who was healthy other than obesity (BMI of 32), presented following 5 days of malaise with a daily fever of $101^{\circ} \mathrm{F}$. On admission, he tested positive for COVID-19 and was placed on $3 \mathrm{~L}$ of $\mathrm{O}_{2}$ via nasal cannula. Following progressive respiratory failure, the patient was transferred to the ICU on hospital day 3 and intubated on day 4 . The patient continued to deteriorate and was placed on extracorporeal membrane oxygenation (ECMO) on hospital days 7-14. While he was weaned off ECMO and back onto mechanical ventilation, stagnant progress on the ventilator was observed where he remained on $50-60 \%$ oxygen for 2 weeks and continued to test positive for COVID-19. The patient received IVIG at $0.5 \mathrm{~g} / \mathrm{kg}$ on hospital days 30-32 with methylprednisolone, with significant improvement in oxygenation and chest X-ray findings (Fig. 3). Approximately $48 \mathrm{~h}$ after IVIG administration, the patient tested negative for COVID-19. He was removed from mechanical ventilation on day 51 and weaned off oxygen on day 53 . The patient was discharged home ambulating on his own and breathing room air on day 60 .

\section{Case 4}

A 30-year-old male patient with sickle cell disease presented with bone and abdominal pain consistent with sickle cell crisis. However, his hemoglobin was $11.7 \mathrm{~g} / \mathrm{dL}$, inconsistent with crisis. He then tested positive for COVID-19, but his chest X-ray was negative. The patient developed hypoxemic encephalopathy and was transferred to the ICU. He was given remdesivir and corticosteroids and intubated on hospital day 2 . His laboratory assessments at this point indicated a D-dimer of $115,000 \mathrm{ng} / \mathrm{mL}$. The patient received IVIG at $0.5 \mathrm{~g} / \mathrm{kg}$ on hospital days 4 and 5 with 


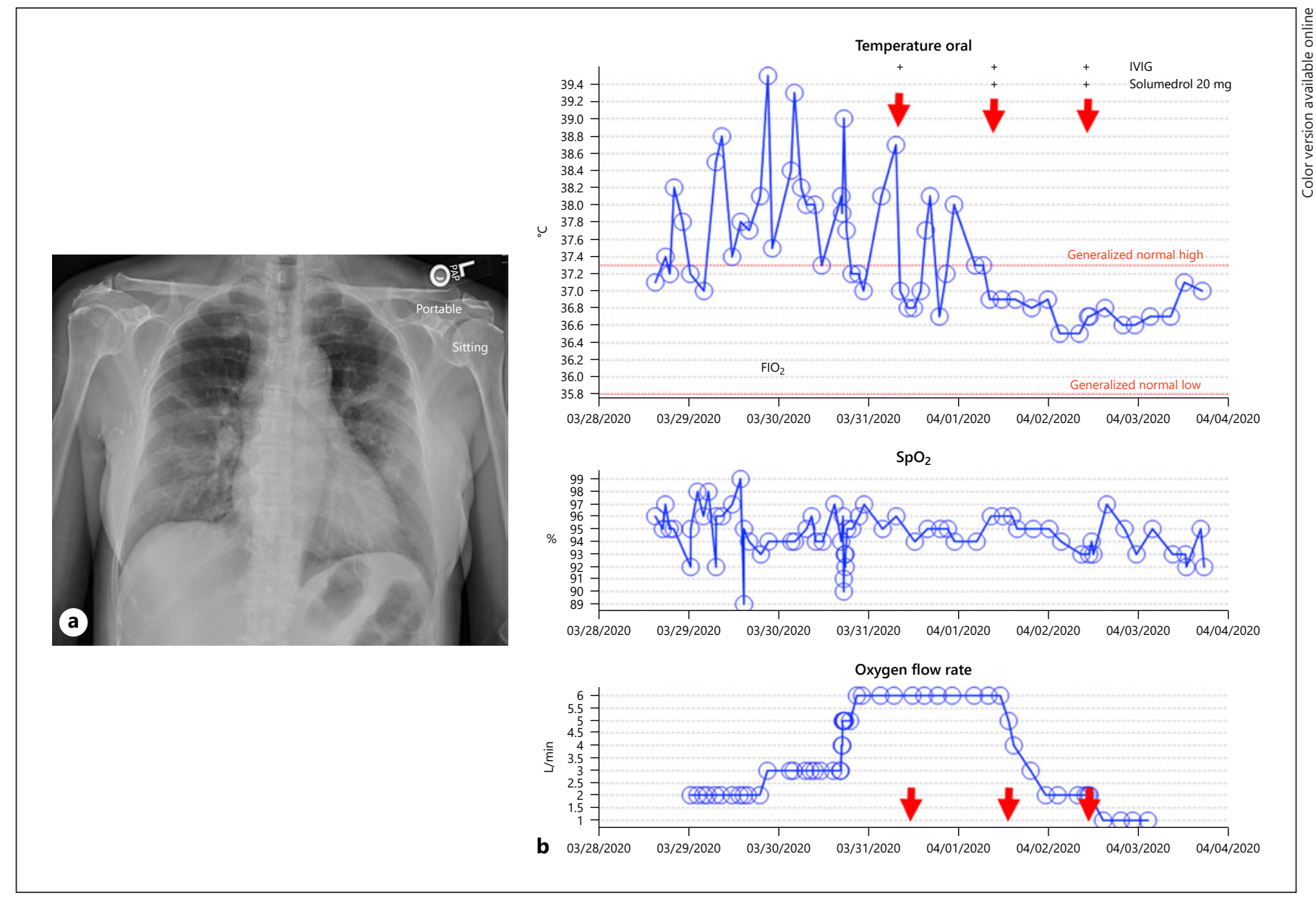

Fig. 2. Clinical details from case 2 including pre-IVIG chest X-ray (a), and vital sign changes in relation to IVIG administration, denoted by the red arrows (b). Top graph shows temperature curve, middle panel the oxygen saturation (\%), and the bottom panel the oxygen flow rate received by the patient over time. IVIG, intravenous immunoglobulin; COVID-19, coronavirus disease 19.
Fig. 3. Chest $\mathrm{X}$-ray images of the COVID-19 patient described in Case 3 in relation to receipt of IVIG therapy, before (a) and after (b), demonstrating rapidly improved aeration. COVID-19, coronavirus disease 19; IVIG, intravenous immunoglobulin
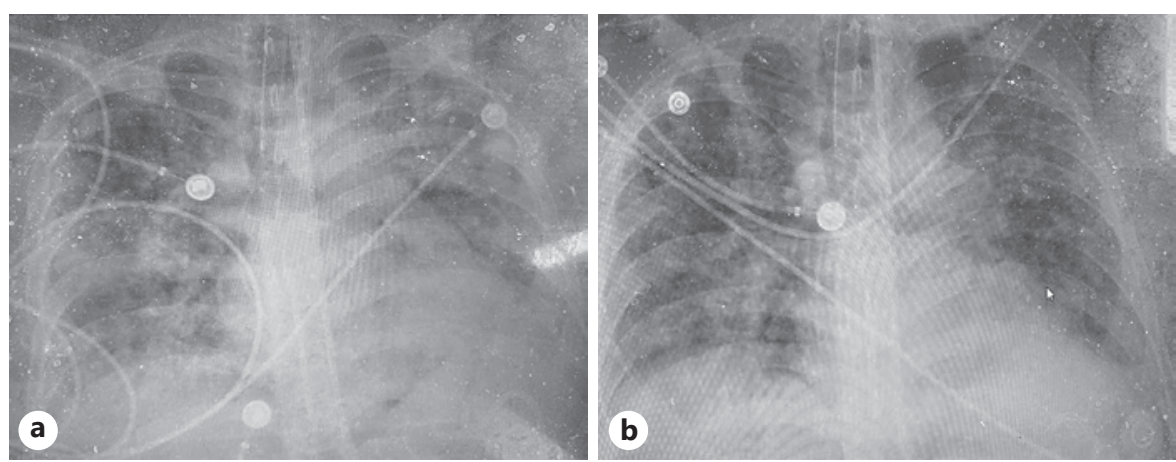

rapid clinical improvement over $48 \mathrm{~h}$ and was extubated (Fig. 4). Approximately 4 days following IVIG administration, his D-dimer decreased to $46,000 \mathrm{ng} / \mathrm{mL}$. He was breathing on room air on hospital day 9 and discharged on hospital day 17 .
Case 5

A 34-year-old woman (G4P2), with a history of asthma, presented with a pregnancy of 26 weeks. She had developed nausea, vomiting, and dyspnea. She was admitted to the hospital 1 week after onset of symptoms and tested positive for COVID-19. The 
Fig. 4. Chest X-ray images of the COVID-19 patient described in Case 4 in relation to receipt of IVIG therapy, before (a) and after (b), demonstrating rapidly improved aeration. COVID-19, coronavirus disease 19; IVIG, intravenous immunoglobulin.

Fig. 5. Chest $\mathrm{X}$-ray images of the COVID-19 patient described in Case 5 in relation to receipt of IVIG therapy, before (a) and after (b), demonstrating rapidly improved aeration. COVID-19, coronavirus disease 19; IVIG, intravenous immunoglobulin.

Fig. 6. Chest X-ray images of the presumed COVID-19 patient described in Case 6 in relation to receipt of IVIG therapy, before (a) and after (b), demonstrating rapidly improved aeration. COVID-19, coronavirus disease 19; IVIG, intravenous immunoglobulin.
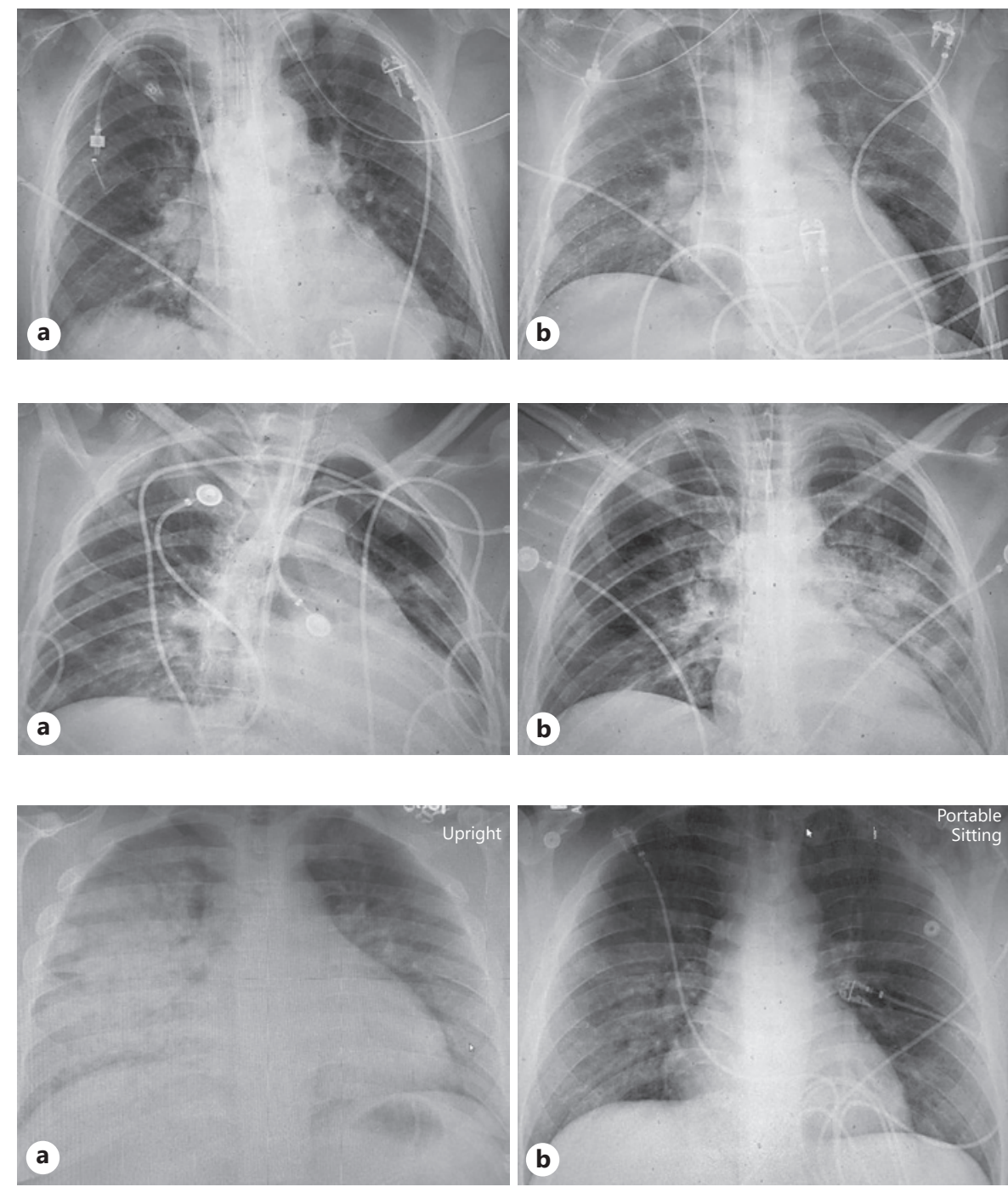

patient was given remdesivir and corticosteroids, but her worsening respiratory symptoms prompted mechanical ventilation on hospital day 4. On hospital day 4, she developed acidosis, hypotension requiring vasopressors, and the fetus showed signs of distress with heart rate decelerations. Betamethasone was administered for fetal lung maturation planning for possible emergent cesarean section. On hospital days 5-7, IVIG was administered at $0.5 \mathrm{~g} / \mathrm{kg}$. On hospital day 6, after 1 dose of IVIG, hypotension and acidosis resolved. She was then weaned off vasopressors, and there were no more signs of fetal distress. Following completion of IVIG therapy, her D-dimers decreased from 3,600 to $900 \mathrm{ng} / \mathrm{mL}$. She also had gradual improvement in oxygenation with improvement in chest X-ray aeration (Fig. 5). The patient was weaned off mechanical ventilation after 11 days, breathing room air 3 days after extubation, and discharged on hospital day 19.

\section{Case 6}

A 23-year-old male without significant medical history was brought in my family for 3 days of gradually worsening confusion, lethargy, and slow speech. Neurological evaluation and neuroimaging were normal, but he was profoundly hypoxemic, quickly requiring a $100 \%$ nonrebreather mask to maintain oxygenation. His chest X-ray showed extensive bilateral infiltrates (Fig. 6). He also had an elevated high-sensitivity troponin of $604 \mathrm{pg} / \mathrm{mL}$ (normal $<19.8$ ), Cr phosphokinase 982 U/L (normal 39-308) with elevated CPK-MB at $9.5 \mathrm{ng} / \mathrm{mL}, \mathrm{D}$-dimer $2,177 \mathrm{ng} / \mathrm{mL}$ (normal $<500$ ), mild increased transaminases, and lymphopenia, with an absolute lymphocyte count of $700 / \mathrm{mm}^{3}$ (lower limit of normal $1,000 / \mathrm{mm}^{3}$ ). A nasopharyngeal swab for severe acute respiratory syndrome coronavirus 2 was negative twice by nucleic acid amplification and by IgG serological testing. An echocardiogram showed mild global hypokinesis with an ejection fraction approximating $50 \%$, without any coronary abnormalities. The patient was given 3 doses of IVIG $0.5 \mathrm{~g} / \mathrm{kg}$ with methylprednisolone $40 \mathrm{mg}$ intravenously $30 \mathrm{~min}$ preinfusion, with very rapid improvement in oxygenation; he was breathing room air in less than $72 \mathrm{~h}$ with improvement in his chest $\mathrm{X}$-ray (Fig. 6). His laboratory values improved, including a CRP decline from 191 to $66 \mathrm{mg} / \mathrm{L}$, troponin decline from 604 to $126 \mathrm{pg} /$ 
$\mathrm{mL}$, and D-dimer decline from 2,177 to $1,342 \mathrm{ng} / \mathrm{mL}$. He was discharged home after 4 days in the hospital.

\section{Case 7}

A 64-year-old male without significant past medical history was transferred to San Diego from Imperial County, California, for higher level of care secondary to severe COVID-19 viral pneumonia. On the day of transfer, he was placed on mechanical ventilation and required vasopressor support. Unfortunately, he sustained barotrauma with pneumomediastinum and bilateral apical pneumothoraces requiring chest tubes. He continued to deteriorate clinically and on hospital day 6 was placed on venovenous ECMO for 14 days. His ensuing hospital course was complicated by Enterococcus faecalis bacteremia, Acinetobacter pneumonia, and Candida parapsilosis fungemia. He received a tracheostomy on hospital day 42 and was transferred from the ICU to the floor. He continued to be COVID-19 positive by nucleic acid amplification for 46 days. The patient's course deteriorated again with an increased oxygen requirement and hypotension, requiring transfer back to the ICU. This was accompanied by a worsening in inflammatory markers with D-dimer increasing from 2,300 to 4,743 $\mathrm{ng} / \mathrm{mL}$ and ferritin 1,181 to $1,774 \mathrm{ng} / \mathrm{L}$ on hospital day 48 . The patient received IVIG at $0.5 \mathrm{~g} / \mathrm{kg}$ on hospital days $49-51$, with methylprednisolone $40 \mathrm{mg}$ IV $30 \mathrm{~min}$ before each infusion. Interestingly, he became COVID-19 test negative on hospital day 53, improved clinically, and was transferred back to the medical floor. After IVIG, his D-dimer decreased to $1,921 \mathrm{ng} / \mathrm{mL}$, CRP decreased from 72 to $9.8 \mathrm{mg} / \mathrm{L}$, and ferritin was stable at $1,794 \mathrm{ng} / \mathrm{mL}$. He was discharged to a rehabilitation facility on hospital day 56.

\section{Case 8}

A 74-year-old woman with no reported comorbidities presented following onset of COVID-19 symptoms for 2 days with a positive test. On admission, she was initially given oxygen support and antibiotics. However, the patient's condition rapidly deteriorated. She was moved to the ICU on hospital day 1 , intubated, and placed on mechanical ventilation and ECMO. The patient was given antibiotics on admission followed by remdesivir on hospital day 7 . The patient's condition continued to worsen. On hospital day 32, she was given IVIG for 4 days at $0.2 \mathrm{~g} / \mathrm{kg}$. Following IVIG administration, the patient's condition improved. She was extubated on hospital day 39 . She was discharged approximately 2 weeks later.

\section{Case 9}

A 68-year-old male with obesity, diabetes mellitus, hypertension, coronary artery disease, and chronic obstructive pulmonary disease (Charlson Comorbidity Index of 6) presented following onset of COVID-19 symptoms for 7 days with a positive test. On admission, he was initially given oxygen support and antibiotics. On hospital day 3, the patient's condition deteriorated. He was moved to the ICU, intubated, and placed on mechanical ventilation and ECMO. The patient was given antibiotics on admission followed by tocilizumab on hospital day 5 . He had also been taking an ace inhibitor for 4 years that was continued during his hospital stay. The patient's condition continued to worsen. On hospital day 9 , he was given IVIG for 3 days at $0.2 \mathrm{~g} / \mathrm{kg}$. At IVIG administration, the patient had an APACHE II Score of 28. Following IVIG administration, the patient's clinical course improved. He was extubated 5 days following IVIG therapy. He continued to improve and was discharged approximately 1 month later.

IVIG for COVID-19
Case 10

A 57-year-old male with obesity, hypertension, coronary artery disease, and chronic obstructive pulmonary disease (Charlson Comorbidity Index of 7) presented following onset of COVID-19 symptoms for 8 days with a positive test. On admission, he was started on oxygen support and antibiotics. On hospital day 3 , the patient was also given lopinavir for 5 days. He had also been taking an angiotensin type- 1 receptor antibody for 2 years that was continued during his hospital stay. Due to his worsening condition, he was moved to the ICU on hospital day 3 and placed on mechanical ventilation. On hospital day 15, he was given IVIG for 4 days at 0.2 $\mathrm{g} / \mathrm{kg}$. At IVIG administration, the patient had an APACHE II Score of 27. Following IVIG administration, the patient's clinical course slowly improved. He was extubated 12 days following IVIG therapy. He continued to improve and was discharged approximately 1 month later.

\section{Case 11}

A 72-year-old male with asthma and coronary artery disease presented following onset of COVID-19 symptoms for 9 days with a positive test. On admission, he was given oxygen support, but his condition rapidly deteriorated. He was moved to the ICU and placed on mechanical ventilation and ECMO. The patient's condition continued to worsen. On hospital day 10, he was given IVIG for 3 days at $0.2 \mathrm{~g} / \mathrm{kg}$. Following IVIG administration, the patient's clinical course slowly improved. He was extubated approximately 10 weeks following IVIG therapy. He continued to improve and was discharged approximately 4 weeks after extubation.

Case 12

A 33-year-old male with no reported comorbidities presented following onset of COVID-19 symptoms for 7 days and a positive test. Following admission, he was given oxygen support and antibiotics. Despite these interventions, the patient's condition deteriorated. To potentially prevent the need for mechanical ventilation, the patient was given IVIG for 4 days at $0.5 \mathrm{~g} / \mathrm{kg}$ on hospital days 5-8. Following IVIG administration, the patient's clinical course rapidly improved. He was able to breathe on room air 1 day following IVIG therapy on hospital day 10 . He was discharged on hospital day 12 .

\section{Discussion/Conclusions}

This small retrospective case series provides a granular view of 12 cases (11 confirmed, 1 highly suspected) of COVID-19 infection resulting in moderate to severe hypoxia treated with IVIG. In all of these cases, IVIG appeared to improve the clinical course, in some instances with immediate and dramatic improvements demonstrated by clinical symptomatology, chest imaging, and laboratory values. These patients demonstrated an extremely high severity of illness with median and mean APACHE II Scores of 13 and 16, respectively, with 5 of 12 requiring ECMO, and yet all 12 patients survived hospitalization. 
The total IVIG dose ranged from $0.5 \mathrm{~g} / \mathrm{kg}$ to $2.0 \mathrm{~g} / \mathrm{kg}$ (median $1.25 \mathrm{~g} / \mathrm{kg}$ ) distributed over 1-4 daily doses. The most common regimen received was $0.5 \mathrm{~g} / \mathrm{kg}$ daily for 3 days. The median time of IVIG administration was 9 days (range 0-48 days) after admission. The median time from first IVIG dose administration to hospital discharge was 14 days (range $3-48$ ). The 5 patients who received IVIG $<4$ days of admission demonstrated a significantly shorter length of hospital stay after treatment (median 7 days, range 3-14 days) than the 7 patients who received it $>7$ days after admission (median 33 days, range 8-48 days, $p=0.03$, Mann-Whitney $\mathrm{U}$ test).

While benefit of IVIG may be greatest when administered early, several of these cases demonstrated improvement after weeks of hospitalization. Also, of great interest were 2 patients with a prolonged and protracted illness with continued COVID-19 (identified by nucleic acid amplification for several weeks). After receiving IVIG treatment, these patients improved enough to be discharged from the hospital shortly thereafter and also tested negative for COVID-19 immediately after treatment.

While definitive, effective treatment of COVID-19 is still lacking, the potential benefit of IVIG in COVID-19 and associated disorders appears to be materializing in several published reports, including in cases of vasculitis and multi-organ injury in the presentation of COVID-19 as a Kawasaki-like disease in children [15-22]. These 12 cases add additional evidence of IVIG benefit in COVID-19, albeit small. More data will soon be available, first of a pilot randomized open-label study of 33 patients comparing IVIG with or without standard of care, and a second more definitive randomized double-blinded placebo-controlled multicenter study performed under the guidance of the US FDA.

With all of the unknowns posed by the COVID-19 pandemic, the data suggesting that IVIG might be beneficial in COVID-19 seem to provide some level of reassurance to clinicians given its widely appreciated use and safety profile over decades. This contrasts to less familiar immunomodulators used by specialists in oncology and rheumatology or other agents that are altogether unapproved for clinical use at this point. Furthermore, given immune system redundancies, IVIG appears to offer global multifaceted mechanisms of immunomodulation to counteract the immune dysregulation (i.e., "cytokine storm") seen in severe COVID-19 infection. Interestingly, as the donor pool serving as the basis if IVIG manufacturing achieves increased immunity over time due to increased exposure to severe acute respiratory syndrome coronavirus 2 , it remains to be determined if additional viral-specific mechanisms will build upon the immunomodulatory ones.

In addition to the small number of cases and that these patients tended to be younger, this study was limited by the fact that no comparator was analyzed. Therefore, despite the immediate and dramatic improvements noted clinically after IVIG administration, it would be hard to definitively prove that IVIG was responsible for the improvement in clinical course.

In summary, we present 12 cases where IVIG appeared to offer benefit to patients with severe COVID-19. More data from prospective studies on the use of IVIG in COVID-19 are highly anticipated in the coming months.

\section{Acknowledgement}

We gratefully acknowledge Shireen Dunwoody of Dunwoody Consulting for her assistance with content organization and development.

\section{Statement of Ethics}

Due to the retrospective nature of this study, waiver of consent/ exempt status was obtained from an Independent Review Board (IntegReview) in the USA and an Ethics Committee in Germany.

\section{Conflict of Interest Statement}

G.S. has received research funding from Octapharma. F.J.F.H. and F.H. have no conflicts of interest to declare.

\section{Funding Sources}

The authors received no funding for this study.

\section{Author Contributions}

G.F.S., F.J.F.H., and F.H. wrote the manuscript and contributed the case data. G.F.S. supervised all steps of the manuscript preparation and is responsible for its scientific content. All authors reviewed and approved the full version of the manuscript. 


\section{References}

1 World Health Organization (WHO) Director-General's opening remarks at the media briefing on COVID-19: 2020 Mar 11. Available at: https://www.who.int/dg/speeches/detail/who-director-general-s-opening-remarks-at-the-media-briefing-on-covid19-11-march- 2020.

2 Johns Hopkins Coronavirus Resources Center. Available at: https://coronavirus.jhu.edu/ map.html.

3 Chen N, Zhou M, Dong X, Qu J, Gong F, Han $\mathrm{Y}$, et al. Epidemiological and clinical characteristics of 99 cases of 2019 novel coronavirus pneumonia in Wuhan, China: a descriptive study. Lancet. 2020;395:507-13.

4 Wang D, Hu B, Hu C, Zhu F, Liu X, Zhang J, et al. Clinical characteristics of 138 hospitalized patients with 2019 novel coronavirus-infected pneumonia in Wuhan, China. JAMA. 2020;323(11):1061-9.

5 Onder G, Rezza G, Brusaferro S. Case-fatality rate and characteristics of patients dying in relation to COVID-19 in Italy. JAMA. 2020; 323(18):1775-6.

6 Richardson S, Hirsch JS, Narasimhan M, Crawford JM, McGinn T, Davidson KW, et al. Presenting characteristics, comorbidities, and outcomes among 5,700 patients hospitalized with COVID-19 in the New York City area. JAMA. 2020;323(20):2052-9.

7 Qin C, Zhou L, Hu Z, Zhang S, Yang S, Tao Y, et al. Dysregulation of immune response in patients with COVID-19 in Wuhan, China. Clin Infect Dis. 2020;71(15):762-8.
8 Mehta P, McAuley DF, Brown M, Sanchez E, Tattersall RS, Manson JJ, et al. COVID-19: consider cytokine storm syndromes and immunosuppression. Lancet. 2020;395(10229):1033-4.

9 Chen X, Zhao B, Qu Y, Chen Y, Xiong J, Feng $\mathrm{Y}$, et al. Detectable serum SARS-CoV-2 viral load (RNAaemia) is closely correlated with drastically elevated interleukin-6 (IL-6) level in critically ill COVID-19 patients. Clin Infect Dis. 2020;71(8):1937-42.

10 Liu Q, Zhou YH, Yang ZQ. The cytokine storm of severe influenza and development of immunomodulatory therapy. Cell Mol Immunol. 2016;13(1):3-10.

11 Ruan Q, Yang K, Wang W, Wang L, Song J. Clinical predictors of mortality due to COVID-19 based on an analysis of data of $150 \mathrm{pa}-$ tients from Wuhan, China. Intensive Care Med. 2020;46(5):846-8.

12 Perez EE, Orange JS, Bonilla F, Chinen J, Chinn IK, Dorsey M, et al. Update on the use of immunoglobulin in human disease: a review of evidence. J Allergy Clin Immunol. 2017;139(3S):S1-46.

13 Afonso AFB, João CMP. The production processes and biological effects of intravenous immunoglobulin. Biomolecules. 2016;6:15.

14 Lee K-Y. Pneumonia, acute respiratory distress syndrome, and early immune-modulator therapy. Int J Mol Sci. 2017;18(2):388.

15 Shao Z, Feng Y, Zhong L, Xie Q, Lei M, Liu Z, et al. Clinical efficacy of intravenous immunoglobulin therapy in critical patients with COVID-19: a multicenter retrospective cohort study. MedRxIV. 2020.
16 Xie Y, Cao S, Dong H, Li Q, Chen E, Zhang $\mathrm{W}$, et al. Effect of regular intravenous immunoglobulin therapy on prognosis of severe pneumonia in patients with COVID-19. J Infect. 2020;81(2):318-56.

17 Zhou AG, Xie SM, Zhang J, Zheng F, Jiang D, $\mathrm{Li} \mathrm{K}$, et al. Short-term moderate-dose corticosteroid plus immunoglobulin effectively reverses COVID-19 patients who have failed low-dose therapy. Preprints. 2020.

18 Cao W, Liu X, Bai T, Fan H, Hong K, Song H, et al. High-dose intravenous immunoglobulin as a therapeutic option for deteriorating patients with coronavirus disease 2019. Open Forum Infect Dis. 2020;7(3):ofaa102.

19 Mohtadi N, Ghaysouri A, Shirazi S, Ansari S, Shafiee E, Bastani E, et al. Recovery of severely ill COVID-19 patients by intravenous immunoglobulin (IVIG) treatment: a case series. Virology. 2020;548:1-5.

20 Jones VG, Mills M, Suarez D, Hogan CA, Yeh D, Segal JB, et al. COVID-19 and Kawasaki disease: novel virus and novel case. Hosp Ped. 2020;10(6):537-40.

21 Verdoni L, Mazza A, Gervasoni A, Martelli L, Ruggeri M, Ciuffreda M, et al. An outbreak of severe Kawasaki-like disease at the Italian epicentre of the SARS-CoV-2 epidemic: an observation cohort study. Lancet. 2020; 395(10239):1771-8.

22 Chiotos K, Bassiri H, Behrens EM, Blatz AM, Chang J, Diorio C, et al. Multisystem inflammatory syndrome in children during the coronavirus 2019 pandemic: a case series. J Ped Infect Dis Soc. 2020;9(3):393-8. 